\title{
OMAE2019-96014
}

\section{AN ACTUATOR DISC ANALYSIS OF A DUCTED HIGH-SOLIDITY TIDAL TURBINE IN YAWED FLOW}

\author{
Mitchell G. Borg \\ Department of Naval Architecture, \\ Ocean, and Marine Engineering \\ University of Strathclyde \\ Glasgow, Scotland, United Kingdom \\ mitchell.borg@strath.ac.uk
}

\author{
Qing Xiao* \\ Department of Naval Architecture, \\ Ocean, and Marine Engineering \\ University of Strathclyde \\ Glasgow, Scotland, United Kingdom \\ qing.xiao@strath.ac.uk
}

\author{
Atilla Incecik \\ Department of Naval Architecture, \\ Ocean, and Marine Engineering \\ University of Strathclyde \\ Glasgow, Scotland, United Kingdom
}

\author{
Steven Allsop \\ Industrial Doctoral Centre for \\ Offshore Renewable Energy (IDCORE) \\ University of Edinburgh \\ Edinburgh, Scotland, United Kingdom
}

\author{
Christophe Peyrard \\ EDF R\&D \\ Electricité de France \\ Research \& Development \\ Chatou, France \\ christophe.peyrard@edf.fr
}

\begin{abstract}
This work elaborates a computational fuid dynamic model utilised in the investigation of the hydrodynamic performance concerning a ducted high-solidity tidal turbine in yawed inlet flows. Analysing the performance at distinct bearing angles with the axis of the turbine, increases in torque and mechanical rotational power were acknowledged to be induced within a limited angular range at distinct tip-speed ratio values. Through multiple yaw iterations, the peak attainment was found to fall between bearing angles of $15^{\circ}$ and $30^{\circ}$, resulting in a maximum power increase of $3.22 \%$, together with an extension of power development to higher tip-speed ratios. In confirmation, these outcomes were subsequently analysed by means of actuator disc theory, attaining a distinguishable relationship with blade-integrated outcomes.
\end{abstract}

\footnotetext{
*Address all correspondence to this author.
}

\section{INTRODUCTION}

Effectively harnessing the power of the ocean for sustainable energy generation is an incredible feat. In effort of increasing the capacity of energy-generating systems, design altercations have been in constant development and assessment, with a plethora attaining implementation within the global market [1]. On the forefront of the pertinent research to push the boundaries of offshore technologies in achieving this endeavour is the increase of mass flow through the turbine, in addition to the alignment the flow, both upstream of the rotor and within the downstream wake. Both objectives of development address increasing the rotor efficiency, and, by extrapolation, the resultant power generated, where the prior objective solely tackles the parameter augmentation regarding one turbine, whereas the latter endeavours in manipulating the dynamics of the domain flow and mitigate efficiency losses within further turbine installations.

As a result of the development attained, two conventional classifications of installations have been implemented within the perimeter of the blades of a turbine: bidirectional ducts and uni- 
directional ducts, illustrated in Figure 1. Both systems have been considered to enhance the performance of a turbine by increasing the flow velocity, together with limiting the spread of the resultant wake, yet their capacity in sustaining sufficient axial flow from a free-stream bearing has not been ascertained for distinct rotor layouts. As a result, the performance capabilities of bidirectional ducts within specific conditions are inadequately understood.

A number of commercial endeavours have attempted to implement ducted-turbine technology to achieve economic prospects, yet a significant quantity have resulted in abandonment [2]. From those prospects, amongst the more substantial, OpenHydro, had achieved commissioning and operation with an open-centre ducted design approach [3,4]. This setup employed an aperture at the centre of the turbine, whilst not only utilising the duct as a flow modifier, but also as a unit in which the power generating system may be housed.

Investigative analyses towards the effectivity of shrouds for the purpose of housing rotors have been directed by numerical methodologies. Werle and Presz [5] presented a theoretical basis, with numerical validation, of the potential improvements available from ducted flow configurations. Modali et al. [6] created a computational fluid dynamic (CFD) model of a horizontal-axis tidal turbine (HATT) in yawed flow to analyse the power generated at different bearing angles. It was acknowledged that at an angle of $15^{\circ}$, power diminished to $70 \%$ of its original power at aligned flow. This highlights the significant loss of transferred energy from the free-stream to the system within acute bearing angles.

Shultz et al. [9] numerically analysed a 2.4 MW turbine within a yaw angle of $-50^{\circ}$ and $50^{\circ}$ at two distinct free-stream velocities. Data showed that the reduction of the power output is systematic to the yaw angle. In addition, the deflection of the wake as a result of the inflow were investigated, where the deflection angles were compared with a modelling approach. A connection line between wake deflection and load asymmetry was deduced, concluding that the results are of particular importance for turbine farm situations with downstream turbines facing the distorted inflow created from upstream ones. Suzuki [10] depicted a similar occurrence in his analysis of twobladed horizontal-axis turbines by numerical means. Within a yaw angle range of $0^{\circ}$ to $45^{\circ}$ a consistent decrease in power was attained with a maximum deficiency of more than $30 \%$ in peak power attainment at a bearing of $45^{\circ}$.

McWilliam et al. [11] proposed a Blade Element Momentum (BEM) model for yawed turbine flows with use of correction factors. Aspects from potential flow methods were implemented within a range of operation conditions within a flow field, attaining better accuracy than conventional BEM methods yet preserving its efficiency. Morote [12] presented a novel method in deriving the effective angle of attack distributions on rotor blades working under swept flow conditions due to yaw angles. The
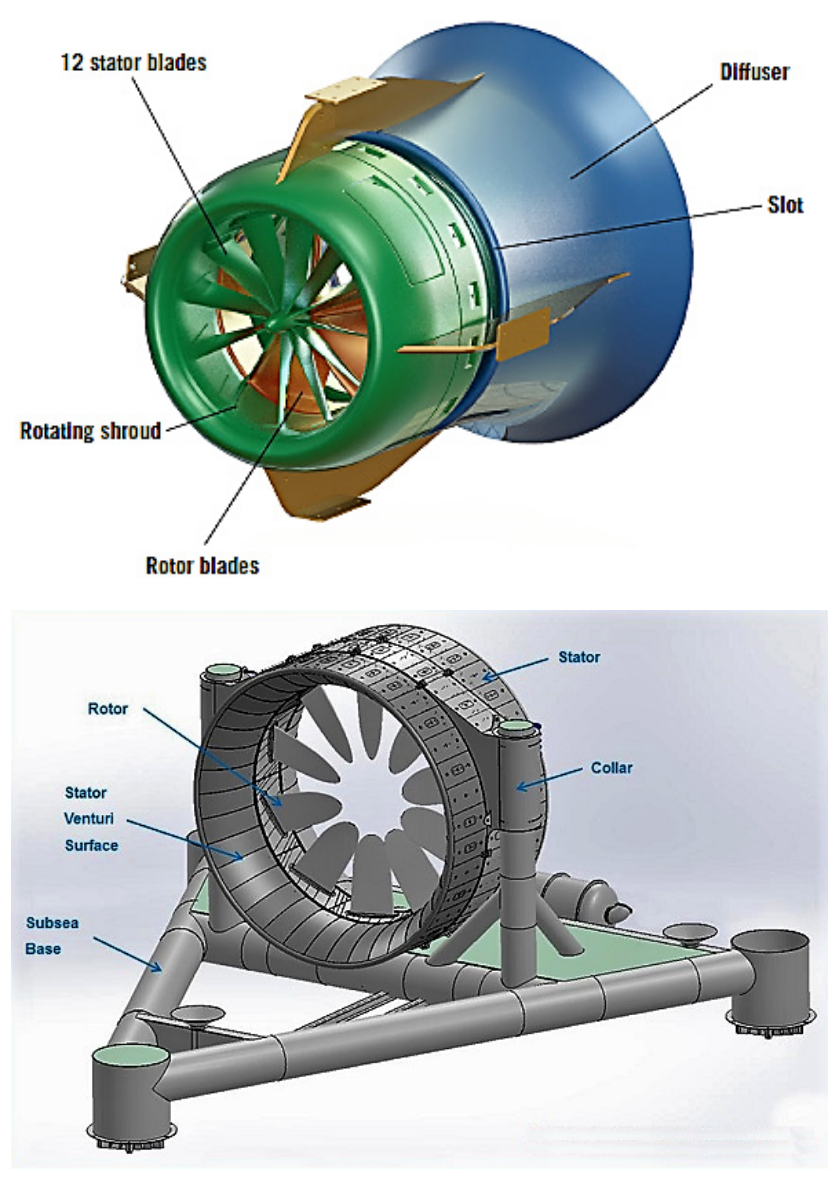

FIGURE 1: Illustrations of uni- (top) [7] and bi- (bottom) [8] directional duct installations

radial flow was coupled to the unperturbed geometric angle, resulting in the projections of the effective angle of attack distributions.

Howland et al. [13] observed wake curling in experiments, which were also reproduced qualitatively in Large Eddy Simulations utilising both actuator disk and actuator line models. Suggested results portrayed that when a turbine is yawed for the benefit of downstream turbines, the curled shape of the wake, and its asymmetry, must be taken into account, due to its effect, dependent on the degree of intersection with downstream turbines.

Fleming and Willden [14] investigated the difference in velocity through the throat of a bidirectional duct in accordance to distinctive duct profiles, where the implemented turbine was represented as an actuator disc utilising CFD. Shives and Crawford [15] analysed a ducted actuator disc model on CFD by varying the angle of attack of a uni-directional duct profile. In continuation, Belloni et al. $[16,17]$ investigated the effects of different bidirectional duct setups utilising a coupled BEM-CFD method, whereas Allsop et al. [18] developed a numerical BEMT model 


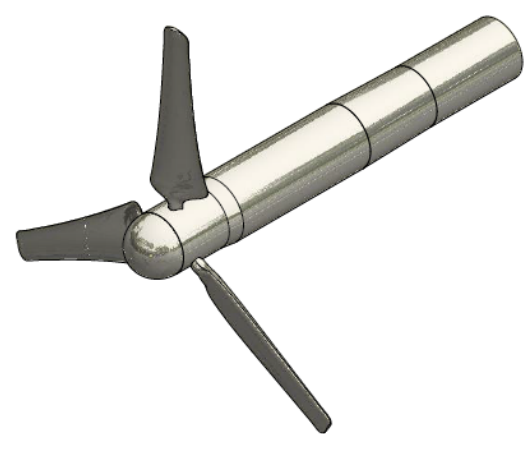

FIGURE 2: Representation of a Three-Bladed HATT [19]

to analyse flow through a duct.

The numerical analysis elaborated in this present study is a continuation to Borg et al. [19], in which a real-scale transient CFD model was developed to assess the hydrodynamic performance of a high-solidity open-centre tidal turbine within a bidirectional duct in aligned flows. This investigation implements a similar aspect, yet within flows at angular bearings, in effort to analyse the hydrodynamic effects of the duct. The individual turbine blades are physically established as explicit geometry, rather than an area-averaged disc, hence replicating their implications in quasi-real-world conditions. The hydrofoil geometry was provided by EDF R\&D to replicate the outcomes of a turbine similar to the design of the OpenHydro PS2 device. In addition, the 7equation RSM (Reynolds Stress Model) turbulence model was coupled to close the Reynolds-Averaged Navier-Stokes (RANS) equation, and analyse the flow domain.

\section{NUMERICAL METHODOLOGY}

The CFD models were computed by means of the commercial solver ANSYS Fluent 16.1, where the physical models were designed to consist of a cuboidal domain layout, imposed with relevant boundary conditions. A Reynolds-Averaged NavierStokes (RANS) turbulence model was implemented in conjunction with time-averaged representations of the continuity and momentum equations which govern the three-dimensional, unsteady, incompressible fluid flow. Periodic conditions were instigated on the planar domain boundaries parallel to the turbine axis and gravitational constant. No-slip wall conditions were induced on the rotor and duct structures, whereas slip conditions were assigned to the remainder of the boundaries of the domain. The domain surrounding the turbine was segregated from the global domain to induce a moving mesh model with rotation at the turbine. Turbulence was modelled by means of the RSM turbulence model due to its superiority in analysing anisotropic flows.

In a similar layout employed by Mason-Jones et al. [20], the dimensions of the computational domain were established to
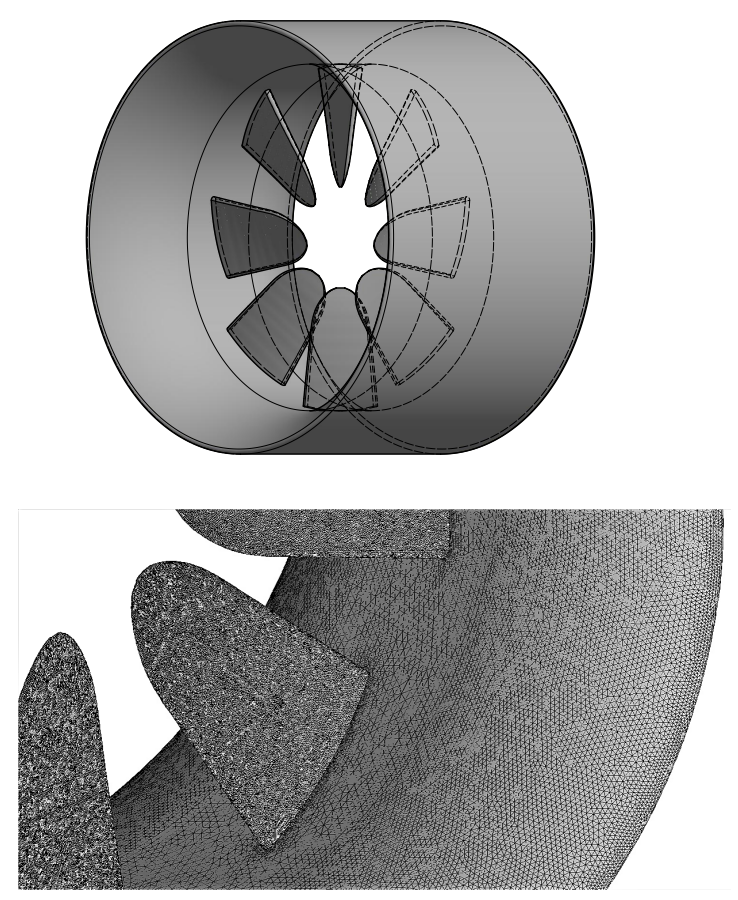

FIGURE 3: (a) Representation of a Ducted High-Solidity OpenCentered Turbine (top); (b) Illustration of the Surface Mesh of the Ducted Turbine (bottom) [19]

attain a maximum numerical blockage ratio of $2 \%$ with the turbine to ascertain the absence of far-field effects and replicate realocean conditions. This corresponded to cross-sectional domain dimensions of $7 D$ at a length of $9 D$, where the inlet and outlet were situated at a distance $3 D$ upstream and $6 D$ downstream of the turbine, respectively.

In an effort to attain a validated CFD model for tidal turbine applications, simulations were established to replicate the experimentation undertaken by Mycek et al. [21]. Identical blade, nacelle, and mast geometry were utilised within the model domain, onto which a tetrahedral mesh was imprinted. The general dimensions of the turbine include a diameter $\mathrm{D}$ of $0.7 \mathrm{~m}$, a nacelle length of $2.5 \mathrm{~m}$, and a mast length of $1 \mathrm{~m}$, as illustrated in Figure 2. The parameters of the turbine and fluid flow were also instated from the literature, with a free-stream velocity of $0.8 \mathrm{~m} / \mathrm{s}$ and an inlet turbulence intensity of $3 \%$.

Upon validation, the model settings, identical to Borg et al. [19], were implemented for yawed-flow analysis of a ducted bidirectional eight-bladed tidal turbine. The general dimensions of the turbine, illustrated in Figure 3a, include a duct diameter of $15 \mathrm{~m}$, rotor diameter of $12 \mathrm{~m}$, and a duct length of $10 \mathrm{~m}$. The turbine geometry was formed within the model domain, onto which a tetrahedral mesh was imprinted. The parameters of the turbine and fluid flow were instated from real-world data, provided 
by EDF R\&D, where three distinct yaw angles were considered: $15^{\circ}, 30^{\circ}$, and $45^{\circ}$ flow, in addition to an aligned flow at $0^{\circ}$.

A mesh independence procedure was carried out on the ducted system by considering the parameter with the highest degree of dynamics, which was the highest TSR condition. Subsequent to the procedure, the final surface mesh count utilised was marginally above 40,000 cells per blade, with dense volumetric cells around the turbine and within the wake, to achieve a total mesh count of 14 million for the ducted turbine. Figure $3 b$ presents an illustration of the ducted turbine surface mesh. The final mesh was then implemented with an appropriate prism layer to achieve a y-plus value of $30 \leqslant y^{+} \leqslant 500$ across the blades and duct.

The CFD computations were performed using the ARCHIEWeSt cluster facility at the University of Strathclyde by running two Intel Xeon Gold $61382.00 \mathrm{GHz}$ computational nodes, with 40 cores and 192 GB of RAM per node per simulation. The ducted turbine simulations were completed within roughly 75 wall-clock hours, equivalent to 3,000 core-hours, hence resulting in 5 wall-clock hours per turbine rotation.

\section{MODEL CHARACTERISATION}

In consideration of the analysis of a physical turbine, notable definitions concerning the resultant performance outcomes, in terms of the boundary conditions employed, are identified. Utilised to attain quasi-real-ocean conditions, the blockage ratio $\left(\alpha_{b l}\right)$ is defined as a correlation between the device reference area $\left(A_{d v c}\right)$ and the domain sectional area $\left(A_{d m n}\right)$ :

$$
\alpha_{b l}=\frac{A_{d v c}}{A_{d m n}}=\frac{\pi R_{d v c}^{2}}{L_{d m n}^{2}}
$$

where $R_{d v c}$ is the device radius, and $L_{d m n}$ is the length of the quadratic cross-sectional area of the computational domain.

The tip speed ratio (TSR) is established as an equivalence between the linear blade-tip velocity and the free-stream velocity:

$$
T S R=\frac{\left|\Omega_{s y s}\right| R_{r t r}}{U_{\infty}}=\frac{\left|\Omega_{x}\right| R_{r t r}}{U_{\infty}}
$$

where $\Omega_{\text {sys }}$ is the system rotational speed, hence $\Omega_{x}$ being the axial angular velocity, $U_{\infty}$ is the free-stream velocity, and $R_{r t r}$ is the rotor radius. A distinction must be made between the device radius and the rotor radius when modelling ducted turbines. In the case of a ducted turbine, $R_{d v c} \neq R_{r t r}$, but $R_{d v c}=R_{d c t}$, contrary to a non-ducted turbine, where $R_{d v c}=R_{r t r}$.

To determine the turbine capacity in converting the fluid free-stream energy into rotational energy, the power coefficient
$\left(C_{P}\right)$ is established. This considers the mechanical rotational power attained by the device $\left(P_{d v c}\right)$ as a ratio of the maximum rotational power potentially acquired in the device area $\left(P_{\infty}\right)$ :

$$
C_{P}=\frac{P_{d v c}}{P_{\infty}}=\frac{M_{x} \Omega_{x}}{\frac{1}{2} \rho A_{d v c} U_{\infty}^{3}}=\frac{M_{x} \Omega_{x}}{\frac{1}{2} \rho \pi R_{d v c}^{2} U_{\infty}^{3}}
$$

where $M_{x}$ is the axial moment, also referred to as the rotor torque.

In relation to the power generated, the torque coefficient $\left(C_{Q}\right)$ evaluates the mechanical torque attained by the device $\left(Q_{d v c}\right)$ as a ratio of the maximum torque potentially acquired in the device area $\left(Q_{\infty}\right)$ :

$$
C_{Q}=\frac{Q_{d v c}}{Q_{\infty}}=\frac{M_{x}}{\frac{1}{2} \rho A_{d v c} R_{r t r} U_{\infty}^{2}}=\frac{M_{x}}{\frac{1}{2} \rho \pi R_{d v c}^{2} R_{r t r} U_{\infty}^{2}}
$$

In ascertaining the forces induced on the turbine, the thrust coefficient $\left(C_{T}\right)$ is established. This considers the drag force on the device $\left(F_{d v c-x}\right)$ as a ratio of the related force on the device area $\left(F_{\infty-x}\right)$ :

$$
C_{T}=\frac{F_{d v c-x}}{F_{\infty-x}}=\frac{F_{d v c-x}}{\frac{1}{2} \rho A_{d v c} U_{\infty}^{2}}=\frac{F_{d v c-x}}{\frac{1}{2} \rho \pi R_{d v c}^{2} U_{\infty}^{2}}
$$

In continuation, the induced axial velocity through the duct $\left(U_{x}\right)$ is compared to the free-stream in an effort to attain a quantitative measure of the capacity of flow alignment to the turbine axis, or the coefficient of axial velocity $\left(C_{U, x}\right)$ :

$$
C_{U, x}=\frac{U_{x}}{\left|U_{\infty}\right|}
$$

Similarly, the pressure difference at the duct $\left(\Delta P_{d c t}\right)$ is compared to the dynamic pressure of the free-stream in an effort to attain a quantitative measure of the capacity of pressure sustainment through the system, or the coefficient of axial velocity $\left(C_{\Delta P_{d c t}}\right)$ :

$$
C_{\Delta P_{d c t}}=\frac{\Delta P_{d c t}}{\frac{1}{2} \rho U_{\infty}^{2}}
$$

\section{RESULTS \& DISCUSSIONS}

Consequent to the methodology, the resultant outcomes, in terms of power coefficient, thrust coefficient, and velocity profiles in the wake, were established for the validation case. As elaborated upon in its previous study [19], the three-bladed HATT CFD model outcomes were compared to the literature [21] introduced above. In comparison to the distinct curves, a total 


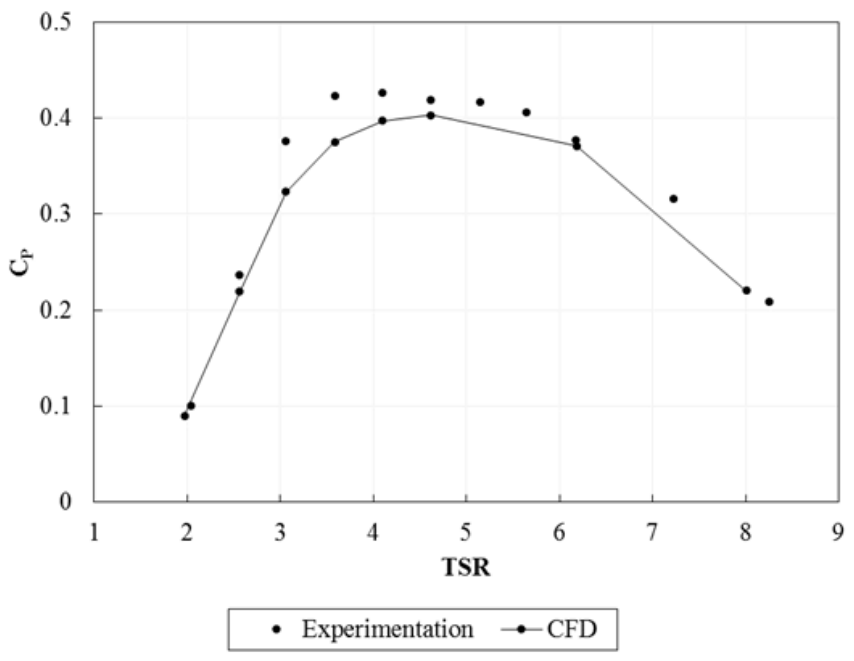

FIGURE 4: CFD to Experimentation Comparison of Power Coefficient with increasing TSR for Three-Bladed HATT [19]

similarity of $95.52 \%$ was acknowledged at the power coefficient plateau region (TSR 3.6 - 6.2), together with all CFD TSR data points falling within $2 \sigma_{C_{P}}$ with experimentation TSR data points, hence within the $95^{\text {th }}$ percentile, displaying good comparison, as illustrated in Figure 4.

In accordance to the validated CFD model, the resultant outcomes, in terms of the power coefficient, torque coefficient, rotor axial velocity, and rotor total pressure difference, were established for all the simulation cases. Literature [16] has speculated the diminishment of the power coefficient of an HATT upon different bearings of yawed flow, resulting in a turbine to become less effective in accordance to the bearing angle. Current data contemplates the effectivity of the installation of a shroud, as a result of an imposed alignment of the flow at the turbine.

Primarily, the resultant power coefficient variation between the yaw angles was established. Illustrated in Figure 5, both the trend and values attained in the power curve vary significantly between aligned flow $\left(0^{\circ}\right)$ and yawed flow. The more notable distinction is the increase in power coefficient at $15^{\circ}$ and $30^{\circ}$ yaw angles within higher TSRs $(1.75-2.5)$, surpassing the maximum power coefficient in aligned flows. In aligned flow, a high gradient increase towards a maximum power coefficient of 0.345 at a 1.75 TSR was acknowledged, followed by a steep decrease. A more steady gradient increase was attained at the different yaw angles, where the nominal TSR shifts to a value of 2 and 2.25 for a bearing of $15^{\circ}$ and $30^{\circ} \& 45^{\circ}$, respectively, with a maximum power coefficient of 0.356 , a $3.2 \%$ increase, attained at a yawed flow of $30^{\circ}$. An increase in global power within the analysed TSR range was also developed, with a $3.02 \%$ increase

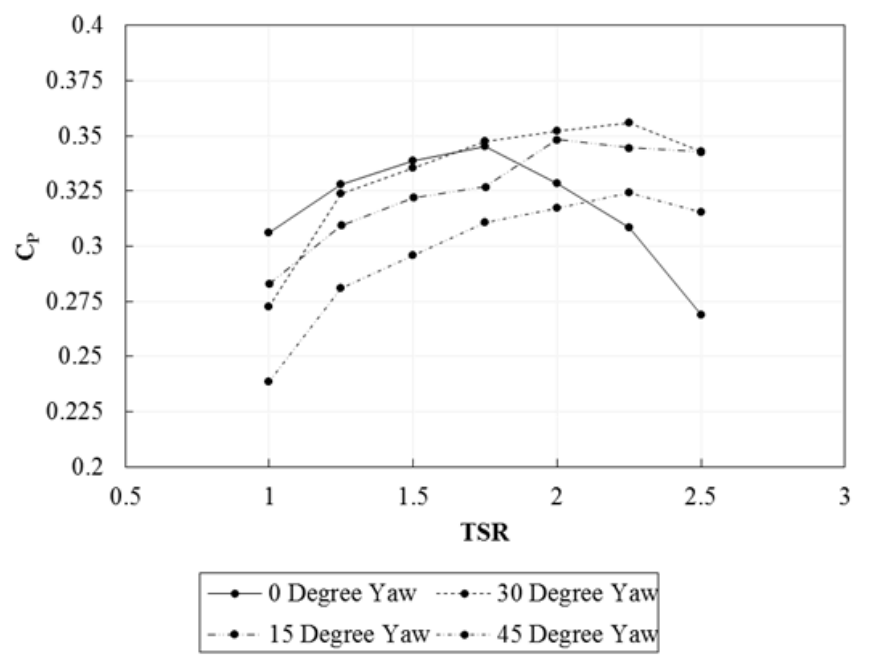

FIGURE 5: Comparison of Power Coefficient with increasing TSR for Ducted Turbine in Aligned and Yawed Flows [19]

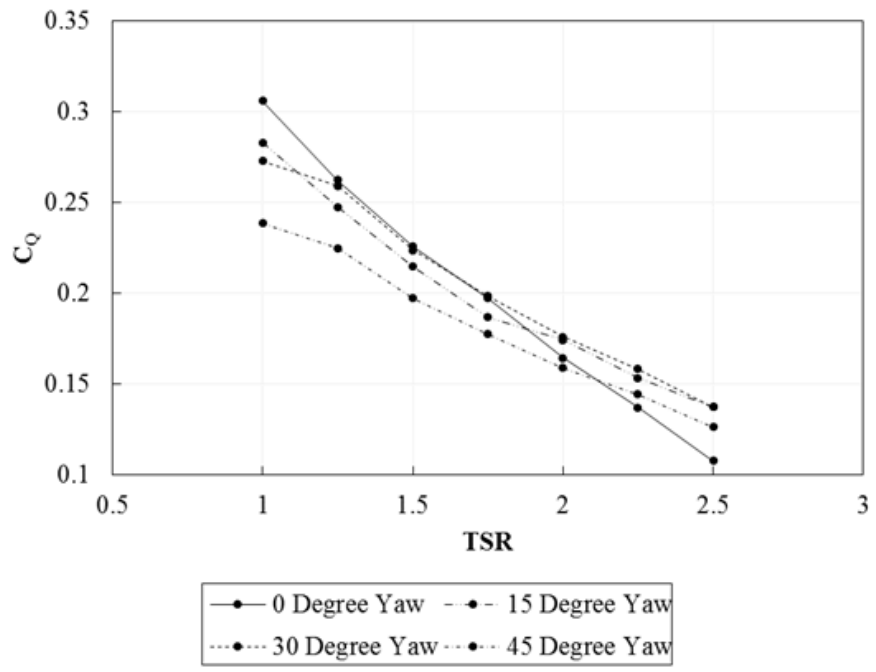

FIGURE 6: Comparison of Torque Coefficient against TSR for Ducted Turbine in Aligned and Yawed Flows

in the integral of the torque coefficient with TSR at a bearing of $30^{\circ}$. Similar to the power coefficient curve, the torque coefficient curve portrays that a higher value was attained at a low TSR for aligned flows, yet acquired substantial terms at high TSRs at a bearing of $30^{\circ}$, as illustrated in Figure 6. 


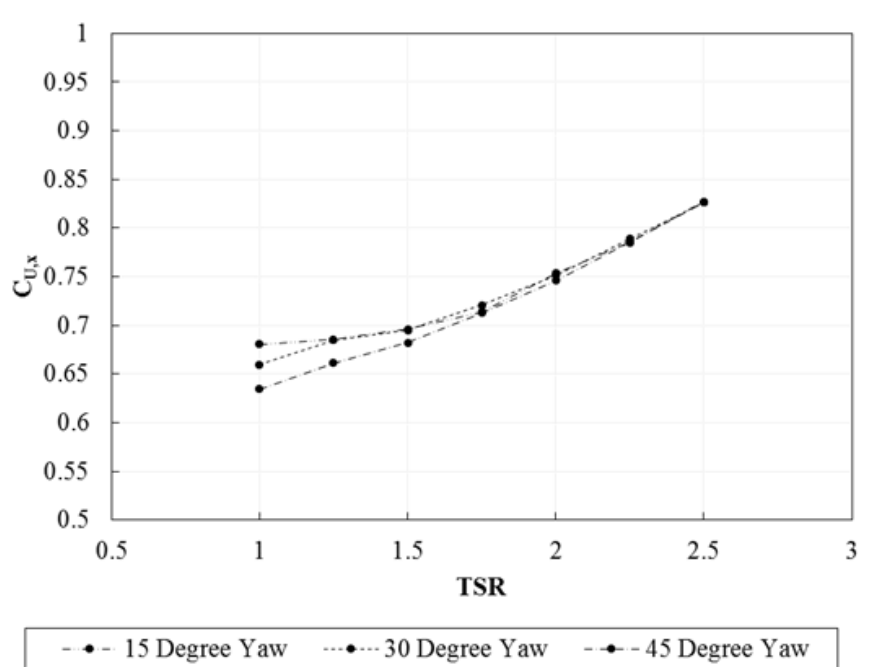

FIGURE 7: Comparison of Axial Velocity Coefficient with increasing TSR at Yawed Flows

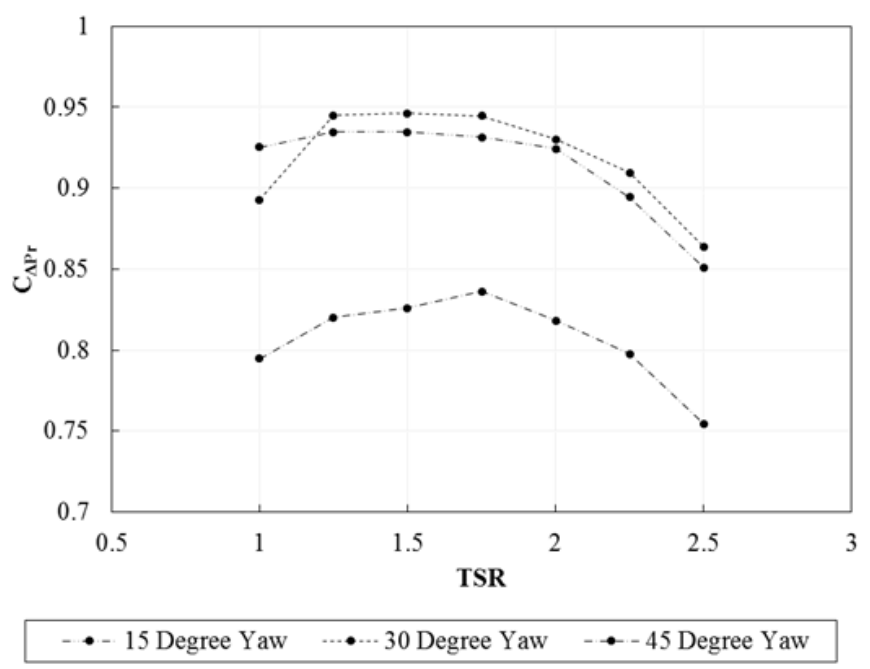

FIGURE 8: Comparison of Total Pressure Difference Coefficient with increasing TSR at Yawed Flows

\section{Actuator Disc Approach}

Momentum theory describes turbine power output within an inviscid flow as a function of the averaged total pressure difference and axial velocity at the turbine, whilst further experimentation has acknowledged that turbulence is also a decisive factor [21]. In investigation of the circumstances for this variation in power, appropriate positions fore and aft of the blade-explicit turbine were selected to analyse the axial velocity and total pres-

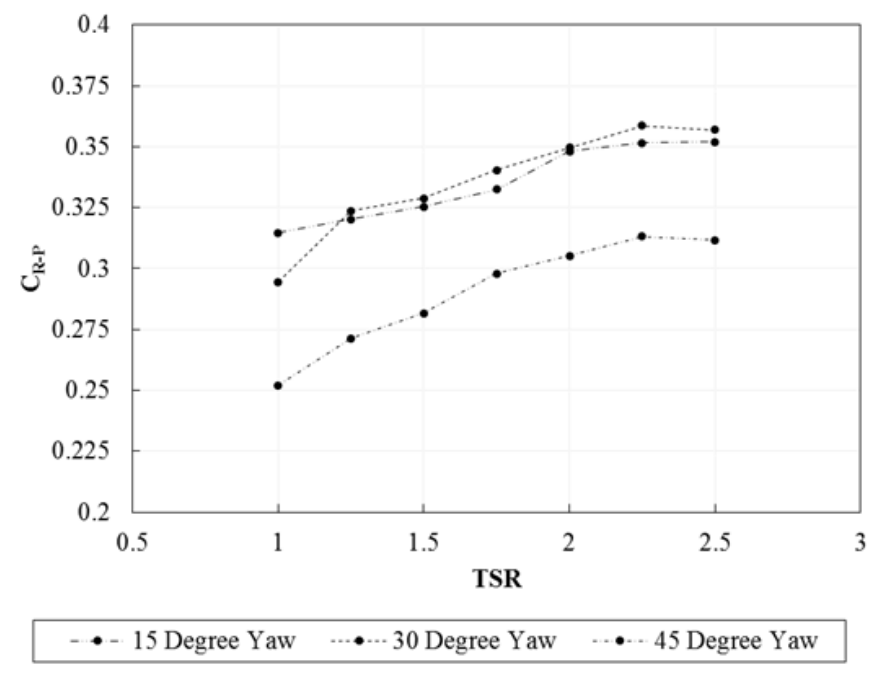

FIGURE 9: Comparison of Representative Power Coefficient $\left(C_{R-P}\right)$ with increasing TSR at Yawed Flows

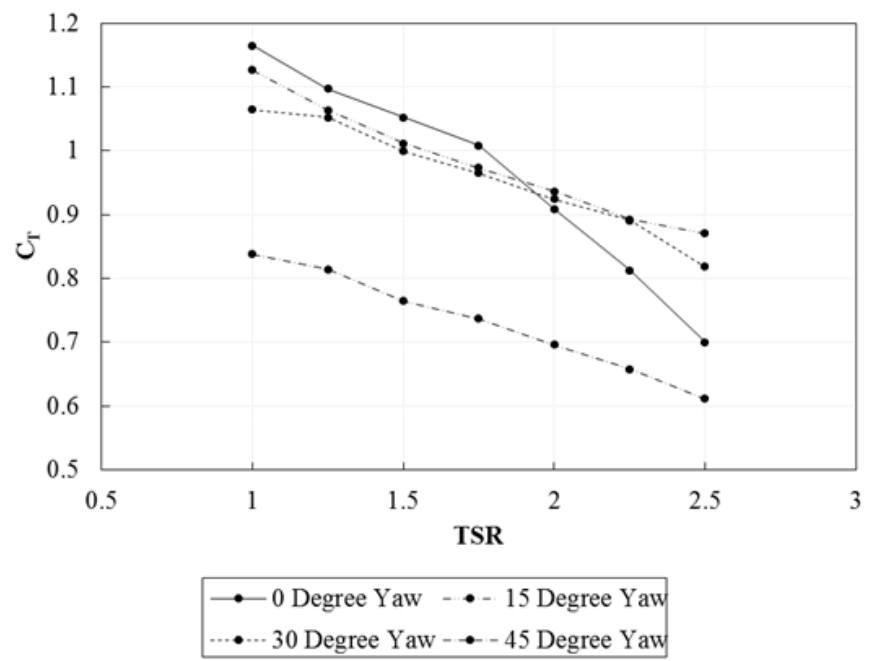

FIGURE 10: Comparison of Thrust Coefficient with increasing TSR at Yawed Flows

sure difference along the turbine rotor in accordance to TSR and yaw angle analysed. Illustrated in Figure 7, the axial velocity of the system at different TSRs is mostly consistent at all different yaw angles, with average values of $\sim 66 \%$ of the free-stream at a TSR of 1 to $\sim 83 \%$ at a TSR of 2.5 . This outcomes portrays the particular capacity of the shroud installation around the rotor in sustaining high degrees of axial velocity through the rotor, albeit with a free-stream angular velocity component variation. 
In continuation, the difference in total pressure fore and aft of the rotor was investigated. Illustrated in Figure 8, the total pressure difference within the system sustains similar trends within different yaw angles, with variations in magnitude, attaining peak values of $\sim 93 \%$ of the free-stream dynamic pressure at a yaw angle of $15^{\circ}, \sim 95 \%$ of the free-stream dynamic pressure at a yaw angle of $30^{\circ}$, and $\sim 85 \%$ at a yaw angle of $45^{\circ}$. The consistent variation in pressure difference stems from the pressures induced upstream and downstream of the duct as a result of the yawed free-stream interaction. Due to the bearing of the flow, the projected area of the inlet within which the flow may proceed diminishes proportionally; in consequence, the static pressure at the inlet lessens accordingly.

A different circumstance occurs at the outlet. As the freestream interacts with the outlet at an angle, the turbulent wake induced by the rotor is propelled aside by the external flow at an angle relative to it. As a result, the back pressure within the duct, and hence the static pressure at the outlet, is significantly reduced, where this variation in pressure at both ends of the duct results in a larger resultant pressure difference across the rotor.

From the notion of actuator disc theory, the coefficients of total pressure difference and axial velocity were considered concurrently as variables within a function, where their product was compared to the power coefficient, as illustrated in Figure 9. By means of the comparison, a significant degree of similarity was attained. Qualitatively, the trend of the turbine powerrepresentation coefficient follows that of the turbine power coefficient; for bearings of $15^{\circ}$, an increase in power within a TSR span of $1.00-2.00$, following a decrease in power at a TSR of 2.25 , whereas at bearings of $30^{\circ} \& 45^{\circ}$, the decrease in power occurs at a TSR of 2.5. Quantitatively, in a comparison between the integral of the power coefficient and that of the representative power coefficient with TSR, an areal similarity of $98.74 \%$, $99.19 \%$, and $98.58 \%$ was attained for yaw angles of $15^{\circ}, 30^{\circ}$, and $45^{\circ}$ respectively, with all data points within the curve attaining more than $92.05 \%$ similarity.

These outcomes, therefore, give evidence of the capacity of a shroud in consideration of a retaining factor in axial velocity, whilst inducing a substantial total pressure difference, to, not only produce higher degrees of mechanical power generation within consistent flows, yet maintains sufficient inlet flow within unsteady domains, and hence, candidates for real-world applications where the inlet flow may vary in bearing.

\section{Thrust Analysis}

Having considered the variation in power generated by means of an increase at distinct yaw angles, together with outcome verification through theoretical actuator disc methodology, the disparity in drag was analysed in accordance to the flow bearing. Illustrated in Figure 10, the depiction ascertains a substantial variation in the force induced upon the turbine system due to the fluid-structure interaction. At aligned flows, the thrust decreases from a substantially high value of 1.16 at low TSR to 1.01 at nominal TSR, at which the gradient of decline increases, to a coefficient of 0.69 at high TSR. Under yawed flows, at bearings of $15^{\circ}$ and $30^{\circ}$, the resultant force decreases in comparison to aligned flows, a result of the misalignment of the flow with a variation of the flow component in the direction of the turbine central axis. The lessened force induction, however, solely acts up to the nominal TSR, after which diminishes, yet sustains a higher value when compared to thrust at aligned flows. This increase in thrust is attributable to the preservation of the pressure difference at high TSRs, which directly generates the drag at the turbine rotor. At a bearing of $45^{\circ}$, the drag forces diminishes substantially in comparison both to aligned and yawed flow. At this angle, the flow component parallel to the rotor axis has halved in magnitude, hence inducing lower thrust, yet introducing resultant lift and heave.

\section{Wake Analysis}

Albeit the pertinent advantageous aspect a duct imposes on a rotor at yawed flow, the developed wake, and its subsequent effect on the general flow, however, may impose secondary disadvantages in the power generated by further turbines at site. Qualitatively illustrated in Figure 11, pressure stagnation points are present at the leading duct ridges at a yaw angle of $15^{\circ}$, which shift towards distinct surfaces of the duct adjacent to the flow bearing. Due to this shift at higher bearing angles, higher magnitudes of pressure are induced at the outer duct surface primarily impacted by the flow (starboard side), and at the inner duct surface on port side, within higher angles of $30^{\circ}$ and $45^{\circ}$. In effect, the opposite oriented surfaces of the duct succumb to regions of substantially lower pressure, inducing suction, as a result of the physical constraint of the structure presence within the flow, emanating a general heave force towards port.

In consequence, the qualitative wake profiles, illustrated in Figure 12 attain a quasi-consistent velocity distribution at the duct region impacted primarily, with consistent flow acting along the outer duct. Within the duct, the velocity is decreased from the free-stream magnitude at the ridges as the static pressure increases, where, in effect of Venturi flow, the magnitude increases to that comparable to the free-stream. At higher flow bearings, an acceleration of flow is present at the inner curved duct surface as a result of the sudden variation in geometry. Further to the physicality of the duct, substantial regions of low-pressure, recirculating flow are present at the port side of the turbine, inducing high degrees of turbulence within the wake. The reduction in static pressure and axial velocity depict detrimental fluid effects within its domain, and hence, within a turbine farm, diminishing the energy potential provided by the conditions of the site. 


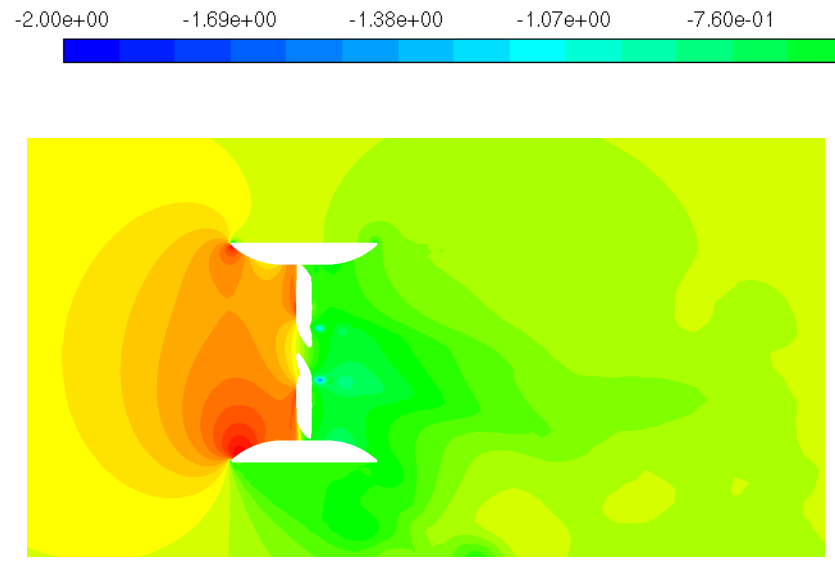

(a) $15^{\circ}$

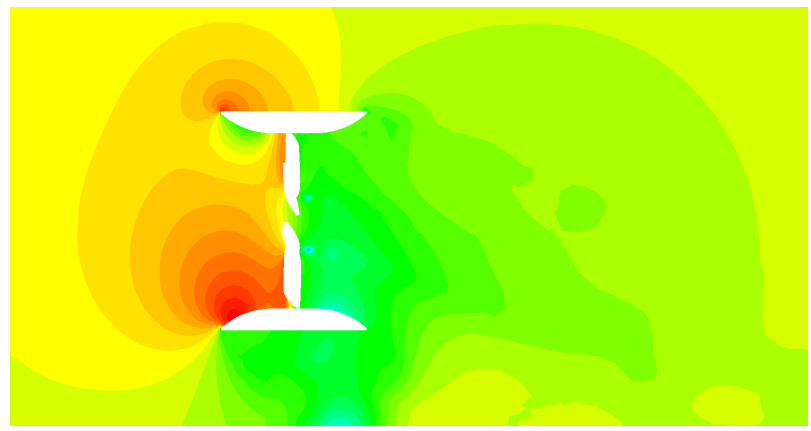

(b) $30^{\circ}$

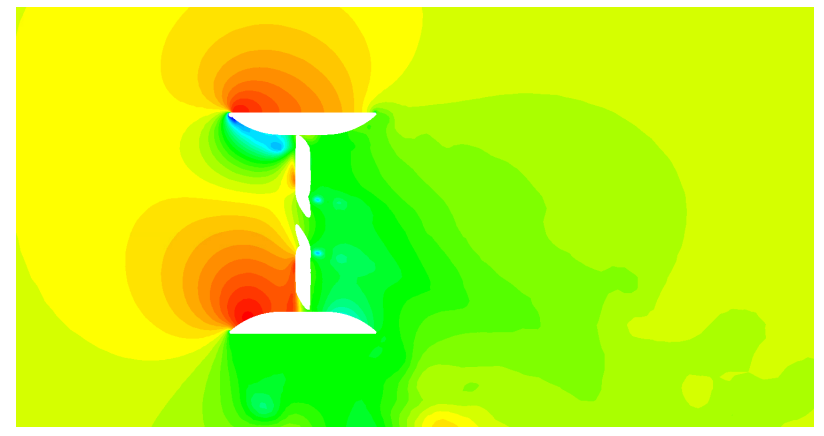

(c) $45^{\circ}$

FIGURE 11: Pressure coefficient contour representation of the domain at distinct flow bearings

\section{CONCLUSION}

Efforts in increasing the efficiency of energy-generating turbines will persevere with pushing the boundaries of offshore technologies. This study has put forward the concept of a computational fluid dynamic analysis of a ducted high-solidity opencentre tidal turbine in an effort to establish its hydrodynamic properties in yawed flow. In continuation to previous analyses regarding the performance in aligned flow, the power and torque output, thrust induction, axial velocity, and total pressure difference fore and aft of the rotor were established. The outcomes depicted both an increase and a shift in the peak power coefficient, in comparison to aligned flow, attained within the higher TSR range at distinct yaw angles; uncharacteristic of an open rotor system.

This notable outcome was evident at flow yaw angles of $15^{\circ}$ and $30^{\circ}$, yet trailed off at a yaw angle of $45^{\circ}$. In the analysis of the rotor by means of actuator disc theory, it was recognised that this increase in power was a result of both the alignment of the axial flow, and the increase in total pressure difference, through the turbine. The successful alignment of the axial flow is induced by the variation in cross-sectional area at the constraint, and hence, the physical specifications of the duct, whilst the in- crease in pressure differential is led by the free-stream interacting with the turbine wake. Further to the increase in performance at the bearings, sustained outputs were attained at higher TSRs, hence developing higher degrees of power generation within the curve. As a result, the duct capacity along yawed flow distinguishes its superiority with non-ducted turbines with regards to sustained power output through distinct bearing angles. This study, therefore, puts forward a considerably advantageous aspect of installing ducted turbines, particularly within domains where the flow magnitude is relatively consistent, yet the direction is significantly fluctuant.

\section{ACKNOWLEDGMENT}

The research work disclosed in this publication is partially funded by the Endeavour Scholarship Scheme (Malta). Scholarships are part-financed by the European Union - European Social Fund (ESF) - Operational Programme II - Cohesion Policy 20142020: "Investing in human capital to create more opportunities and promote the well-being of society".

Results were obtained using ARCHIE-WeSt High Performance Computer (www.archie-west.ac.uk). 


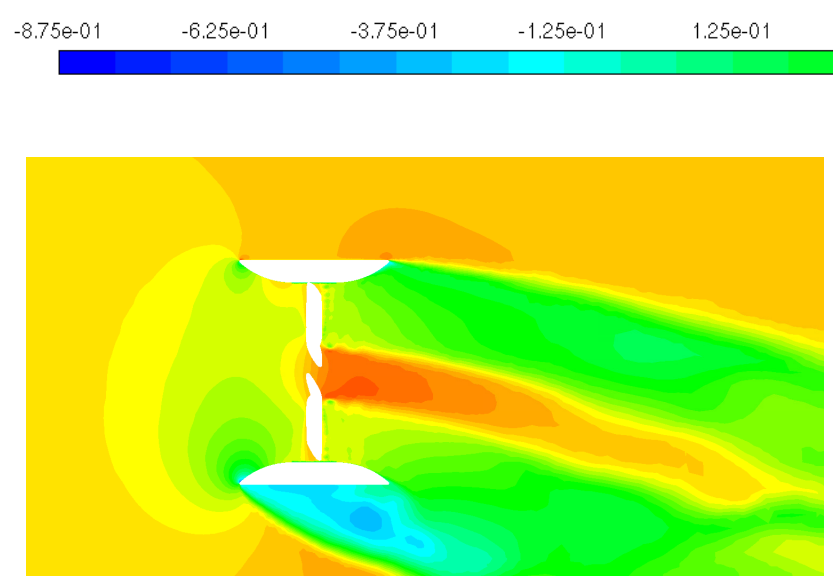

(a) $15^{\circ}$

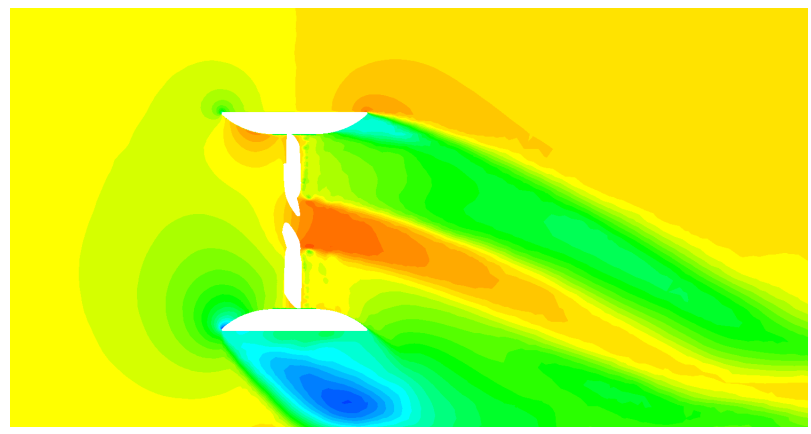

(b) $30^{\circ}$

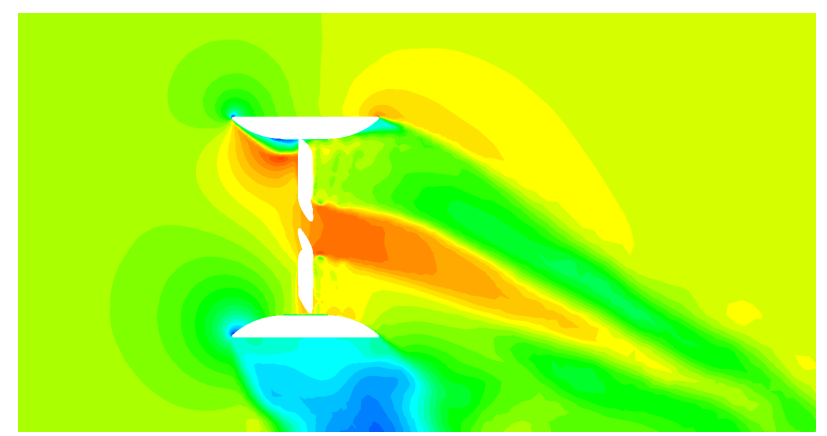

(c) $45^{\circ}$

FIGURE 12: Axial velocity coefficient $\left(C_{U, x}\right)$ contour representation of the domain at distinct flow bearings

\section{REFERENCES}

[1] O Rourke, F., Boyle, F., and Reynolds, A., 2010. "Tidal energy update 2009”. Applied Energy, 87(2), pp. 398-409.

[2] Gipe, P., 2018. WIND-WORKS: Ducted Wind Turbines.

[3] Bloomberg L.P., 2018. OpenHydro Group Limited: Private Company Information - Bloomberg.

[4] OpenHydro Group Ltd., 2016. Projects.

[5] Werle, M. J., and Presz, W. M., 2008. "Ducted Wind/Water Turbines and Propellers Revisited". Journal of Propulsion and Power, 24(5), 9, pp. 1146-1150.

[6] Modali, P., Kolekar, N. S., and Banerjee, A., 2018. "Performance and Wake Characteristics of a Tidal Turbine under Yaw". International Marine Energy Journal, 1, 8, pp. 4150.

[7] Billett, R., 2015. "Rising Tide". ANSYS ADVANTAGE, IX(3), pp. $25-27$.

[8] Crist Offshore, 2015. Open-Centre Tidal Turbine.

[9] Schulz, C., Letzgus, P., Lutz, T., and Kramer, E., 2016. "CFD study on the impact of yawed inflow on loads, power and near wake of a generic wind turbine". Wind Energy, 20, 7, pp. 253-268.

[10] Suzuki, M., 2012. "Numerical Analysis of Horizontal-Axis
Wind Turbine Characteristics in Yawed Conditions". Open Journal of Fluids Mechanics, 2, 12, pp. 331-336.

[11] McWilliams, M. K., Lawton, S., Cline, S., and Crawford, C., 2011. "A Corrected Blade Element Momentum method for Simulating Wind Turbines in Yawed Flow”. In 49th AIAA Aerospace Sciences Meeting including the New Horizons Forum and Aerospace Exposition, AIAA.

[12] Morote, J., 2015. "Angle of attack distribution on wind turbines in yawed flow". Wind Energy, 19, 6, pp. 681-702.

[13] Howland, M. F., Bossuyt, J., Martinez-Tossas, L. A., Meyers, J., and C., M., 2016. "Wake structure in actuator disk models of wind turbines in yaw under uniform inflow conditions". Journal of Renewable and Sustainable Energy, 1, 6, pp. 1-17.

[14] Fleming, C. F., and Willden, R. H., 2016. "Analysis of bidirectional ducted tidal turbine performance". International Journal of Marine Energy, 16, pp. 162-173.

[15] Shives, M., and Crawford, C., 2012. "Developing an empirical model for ducted tidal turbine performance using numerical simulation results". Proceedings of the Institution of Mechanical Engineers, Part A: Journal of Power and Energy, 226(1), 2, pp. 112-125. 
[16] Belloni, C. S., Willden, R. H., and Houlsby, G. T., 2013. "A Numerical Analysis of Bidirectional Ducted Tidal Turbines in Yawed Flow". Marine Technology Society Journal, 47(4), 7, pp. 23-35.

[17] Belloni, C., Willden, R., and Houlsby, G., 2017. "An investigation of ducted and open-centre tidal turbines employing CFD-embedded BEM". Renewable Energy, 108, 8, pp. 622-634.

[18] Allsop, S., Peyrard, C., Thies, P. R., Boulougouris, E., and Harrison, G. P., 2017. "Hydrodynamic analysis of a ducted, open centre tidal stream turbine using blade element momentum theory". Ocean Engineering, 141, 9, pp. 531-542.

[19] Borg, M. G., Xiao, Q., Incecik, A., Allsop, S., and Peyrard, C., 2018. "Numerical Analysis of a Ducted High-Solidity Tidal Turbine”. In OCEANS'18 MTS/IEEE Kobe, IEEE.

[20] Mason-Jones, A., O’Doherty, D. M., Morris, C. E., O’Doherty, T., Byrne, C. B., Prickett, P. W., Grosvenor, R. I., Owen, I., Tedds, S., and Poole, R. J., 2012. "NonDimensional Scaling of Tidal Stream Turbines". Energy, 44, pp. 820-829.

[21] Mycek, P., Gaurier, B., Germain, G., Pinon, G., and Rivoalen, E., 2014. "Renewable Energy Experimental study of the turbulence intensity effects on marine current turbines behaviour. Part I: One single turbine”. Renewable Energy, 66, pp. 729-746. 